\title{
Vibration-based SHM strategy for a real time alert system with damage location and quantification
}

\author{
Ana Fernández-Navamuel ${ }^{1}$, Diego Zamora-Sánchez ${ }^{1}$, Tomás Varona-Poncela ${ }^{1}$, Carlos \\ Jiménez-Fernández ${ }^{1}$, Jesús Díez-Hernández ${ }^{1}$, David García-Sánchez ${ }^{1}$ and David \\ Pardo $^{2}$ \\ ${ }^{1}$ TECNALIA, Basque Research and Technology Alliance (BRTA), Parque Científico y \\ Tecnológico de Bizkaia Astondo bidea \\ Edificio 700, E-48160 Derio, Spain. \\ ${ }^{2}$ University of the Basque Country (UPV/EHU), Basque Center for Applied Mathematics \\ (BCAM), and Ikerbasque, Bilbao, Spain
}

\begin{abstract}
We present a simple and fully automatable vibration-based Structural Health Monitoring (SHM) alert system. The proposed method consists in applying an Automated Frequency Domain Decomposition (AFDD) algorithm to obtain the eigenfrequencies and mode shapes in real time from acceleration measurements, allowing to provide a diagnosis based on a Support Vector Machine algorithm trained with a database of the modal properties in undamaged and damaged scenarios accounting for temperature variability. The result is an alert system for controlling the correct performance of the structure in real time with a simple but efficient approach. Once the alert is triggered, the undamaged mode shapes (which could be previously stored in a database of modal parameters classified by temperature) and the current (damaged) mode shapes, can provide guidance for further application of Finite Element Model Updating (FEMU) techniques. The method is trained and validated with simulations from a FE model that is calibrated employing a genetic algorithm with real data from a short-term vibration measurement campaign on a truss railway bridge in Alicante (Spain).
\end{abstract}

Keywords: Structural Health Monitoring, Structural Dynamics, Bridge Maintenance, Machine Learning.

\section{Introduction}

Structural Health Monitoring (SHM) is a multi-disciplinary procedure for the evaluation and control of the state of real structures [1]. With the latest improvements in the areas of instrumentation, data acquisition and transmission, a door has been opened in this field thanks to the possibility of disposing of large amounts of data without excessive costs [2,3]. Hence, research interests in this area are now posed on the development of powerful algorithms that can receive and interpret these data and transform them into useful information to help managers in decision making [1]. 
In this sense, one of the most broadly developed methodologies in the field of civil engineering is known as vibration based SHM [3]. This discipline builds on the existing relation between the modal response of a structure and its physical properties such as mass or stiffness $[3,4]$. Since the presence of structural damage directly affects the physical properties, it will then induce changes in the modal response of the considered structure [4].

There are two main challenges for modal identification in real practice. First, precision in the properties must be enough to track the structural response [5]. Second, the selected method should be suitable for automation to display results close to realtime and provide continuous assessment [5,6]. In bridges, where it is not economic to perform Experimental Modal Analysis (EMA) with known loads (shakers, drop weights, etc.), Operational Modal Analysis (OMA) techniques are the standard for vibration-based SHM [5,7]. One of the most commonly employed OMA methodologies is the Frequency Domain Decomposition (FDD), introduced in [8]. This method has the advantage of being easily automatable $[9,10]$. There exist several works in the literature attempting to reach this goal, including the standard Automated Frequency Domain Decomposition (AFDD) [11] and automatic applications of the Stochastic Subspace Identification method (SSI) $[10,12]$.

In the field of SHM, the implementation of damage detection algorithms using the modal response can be addressed from two main perspectives: model-based and datadriven [3]. Model-based approaches mostly rely on Finite Element Model Updating (FEMU), which minimizes the discrepancy between the experimental and the numerical responses by modifying certain parameters in physics-based models [13]. Its main drawback is that it implies solving several direct problems to achieve convergence, which prevents its use from real-time applications [1,3]. Data-driven methods exploit the potential in the data acquired during monitoring campaigns to characterize the possible states of the structure and solve classification problems by associating labels to different scenarios [1]. In addition, once these algorithms have been trained and validated, they can provide fast predictions based on the learned information, thus addressing real-time applications [14]. An important fact when working with real fullscale structures is the effect of operational and environmental conditions in the measured data, which can mask the presence of certain damages and hinder the performance of the algorithms [3]. Hence, dealing with the effect of a changing environment is a key issue in the field, and there exist several works employing different techniques, such as Principal Component Analysis or regression models $[15,16]$.

In addition, experimental data under possible damage scenarios is rarely available in real practice [1]. This lack of information makes the training of these algorithms an Unsupervised Learning task (only information about the undamaged condition is used), leading to achieve only the first Level in the Rytter's scale of damage states [17].

This work addresses the development of a data-driven damage detection algorithm for the evaluation of the state of a steel truss bridge in Alicante (Spain) accounting for the effect of varying environmental conditions. We use the acceleration measurements acquired after train passage recorded during a short-term monitoring experience to extract the modal properties of the structure (representing the baseline condition) and use them to calibrate a FE model of the bridge with a genetic algorithm. Finally, we use 
this model to simulate ambient vibration records at different temperatures and damage scenarios to train a Support Vector Machine (SVM) algorithm for damage detection. We validate the methodology with a testing database that contains new scenarios.

\section{Methodology}

\subsection{Description of the structure}

The structure under study is a steel bridge with a Pratt truss scheme, which serves as a passage over the gulch known as Barranco de Aguas for Line 1 of the FGV TRAM Network in Alicante (Spain). The bridge has a total length of approximately 106 meters, consisting of two sections: one hyperstatic with two spans of 42.00 meters each and another isostatic span of 21.12 meters length, being this last one the chosen to be monitored. This span was chosen because of a fatigue study performed to the previous old bridge, which was then replaced for the current bridge in 2018 with same overall structural scheme (see images in Fig. 1 for the old and new structure).

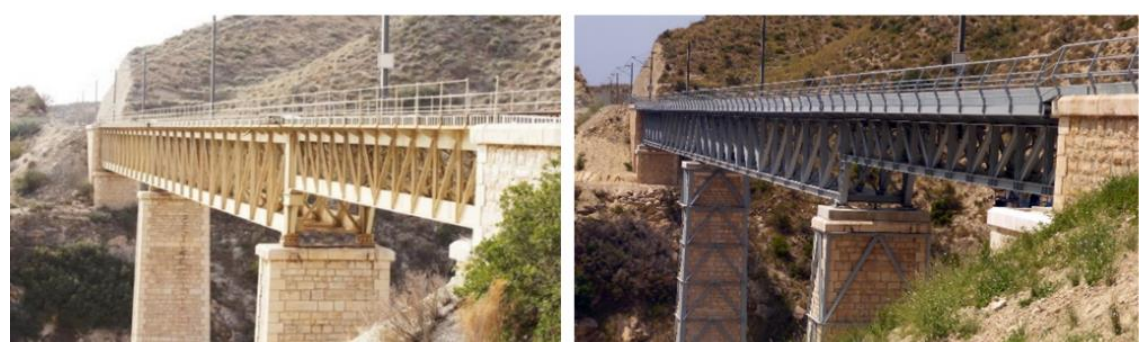

Fig. 1 Old and new bridges over Barranco de Aguas, with trusses of identical scheme and dimensions, but different structural elements

According to the real geometry and material properties of the bridge, a parametric finite element model was built in ANSYS APDL with beam type elements (BEAM188) for the truss steel profiles and shell type elements (SHELL181) for the composite slab and concrete low walls. Despite the span is assumed to be isostatic, it is observed that the analytical response fits better with the measured modes by adding some extra longitudinal restraint given by adjustable elastic springs (LINK180), which are finally added for calibration. Fig. 2 shows the FE model built in ANSYS APDL.

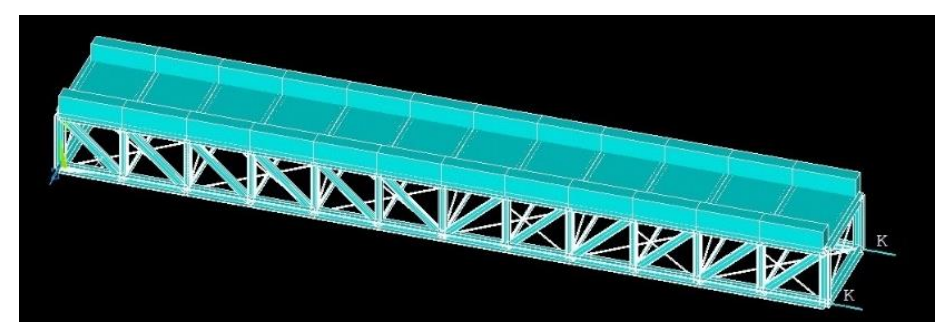

Fig. 2 Detail of the 3D model built in ANSYS APDL for the analyzed span 


\subsection{Modal identification}

In this work, a short-term monitoring period was carried out to record acceleration measurements after train passage on the bridge. The instrumentation system included triaxial accelerometers with different specifications. We employed mainly accelerometers with $\pm 2 \mathrm{~g}$ of full scale for the registration of free vibration measurements occurring once the train has passed the viaduct. Ten accelerometers are placed on the bottom chord (five on each side of the bridge: every two panels), as shown in Fig. 3. In this experience, the duration of the measurements was limited by the sensitivity of the accelerometers. Thus, a high sampling rate $(600 \mathrm{~Hz})$ was employed to compensate the shorten in duration.

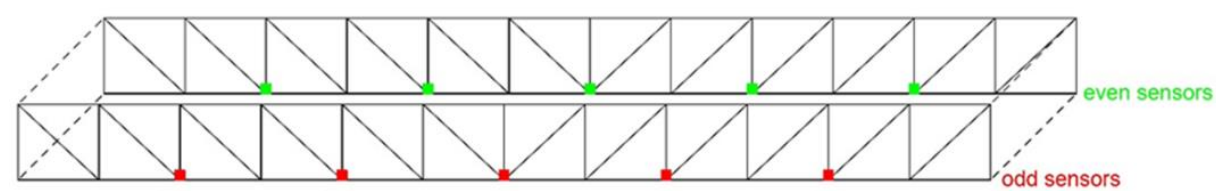

Fig. 3 Scheme and photos showing sensor placement

Since the objective of this work was to provide a close to real-time assessment tool, we use a MATLAB implementation of the already introduced standard Automated Frequency Decomposition (AFDD) to obtain the eigenfrequencies and mode shapes. The methodology is fully explained in $[8,10]$. We use the obtained eigenfrequencies and mode shapes as the input to an updating step to calibrate an initial FE model based on design specifications that will be used for the training and validation of the damage detection algorithm. However, an intermediate step is required before entering the calibration process, since the mode shape vectors are hardy manageable [18]. The Modal Assurance Criterion (MAC) is used as damage sensitive feature to update the FE model [19]. It measures the discrepancy between the corresponding numerical and experimental mode shapes, and can be written as:

$$
M A C=\frac{\left|\phi_{\text {exp }}^{T} \cdot \phi_{\text {num }}\right|^{2}}{\left\|\phi_{\text {exp }}\right\|^{2}\left\|\phi_{\text {num }}\right\|^{2}}
$$

where $\phi_{\text {exp }}$ and $\phi_{\text {num }}$ stand for the experimental and numerical mode shapes, respectively.

In a preliminary analysis, it was found that the second mode (torsion) was close to the first mode (first bending mode), generating convergence problems in the model updating algorithm due to permutation of modes. Therefore, only bending modes are considered in this work, computing the first and third mode shapes only (see the chosen modes in Fig. 4 and Fig. 5). Given the existence of symmetry, only the data of one side of the bridge (five sensors) is processed. The FE model is correspondingly restrained to avoid the appearance of undesirable modes. The calibration algorithm uses four features for updating (the first two bending natural frequencies and the corresponding MAC values). 


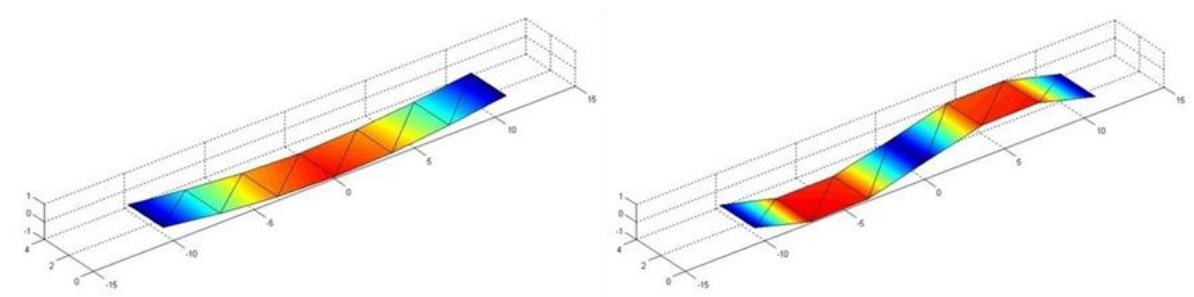

Fig. 4 Detail of the first and third experimental 3D mode shapes (bending modes) obtained with MACEC
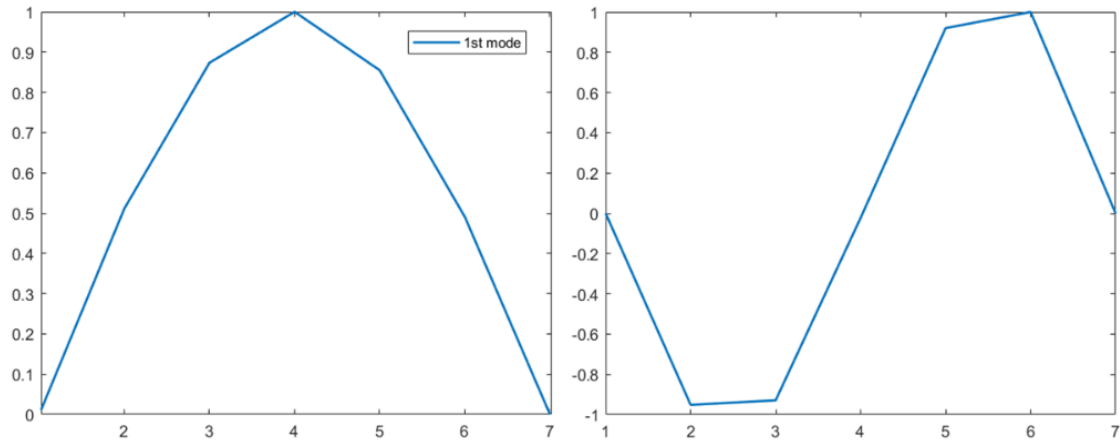

Fig. 5 Detail of the corresponding experimental 2D mode shapes obtained with AFDD

\subsection{Machine Learning algorithm for novelty detection (SVM)}

In this work we propose the implementation of a damage detection tool to identify the presence of abnormal behavior under different environmental conditions based on the dynamic response of the structure. The proposed algorithm is known as Support Vector Machine (SVM), commonly used to solve both classification and regression problems [20,21].

The methodology consists in five main steps: (a) modeling of the effect of temperature, (b) generation of the synthetic damage scenarios and (c) configuration of the SVM algorithm.

\section{a) Modelling the effect of temperature}

Given the structural characteristics of the bridge under study together with its location, the main source of variability during normal service is temperature. Hence, we will account for this phenomenon using the model presented in [22]. This model relates the elastic modulus of steel with temperature and is shown in Fig. 6. The curve can be approximated by the following polynomial of second order:

$$
E_{\text {steel }}(T)=3.51 \cdot 10^{-5} \times T^{2}-3.89 \cdot 10^{-3} \times T+2.03
$$




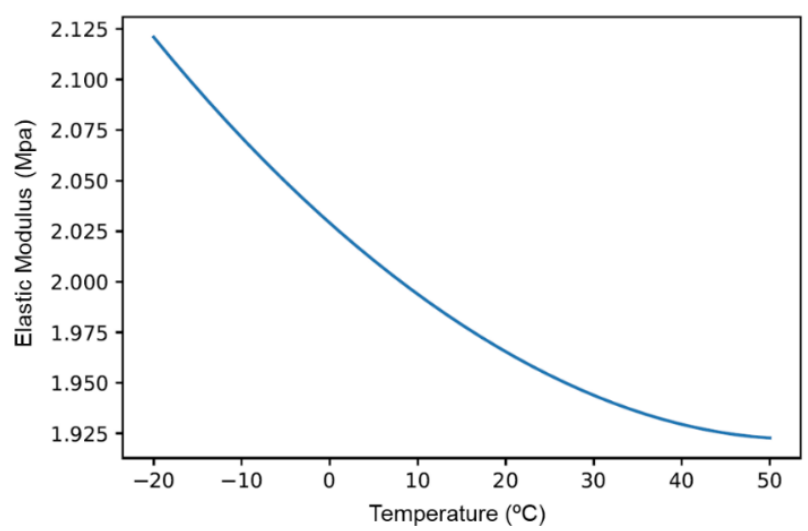

Fig. 6 Steel elastic modulus evolution with temperature [22]

We employ this function to generate a set of models whose response is representative of the undamaged state of the bridge under different ambient temperatures.

\section{b) Generation of the synthetic damage scenarios}

Similar to the temperature effect, we must somehow modify the parameters in the calibrated FE to account for the presence of damage. These synthetic damage regions are found to be enough to give robustness to the algorithm so that it can classify new data and assess the presence of damage. We generate damage distinguishing three different regions along the bridge length, each of them represented by the value of the elastic modulus of the elements belonging to that region. Damage at a certain zone is simulated through a reduction of the undamaged elastic modulus values. Hence, the damage levels are represented by the admissible range of variation with respect to the reference value. In this work, we considered four reference values according to seasonal average temperatures, to apply the reduction factors due to damage. Table 1 shows the corresponding ranges.

Table 1 Damage labels for classification

\begin{tabular}{ccc}
\hline Damage level & Range of values $(\%)$ & Class Label \\
\hline (Undamaged) & Temperature variability only & 1 \\
Slight & $(70-80]$ & 2 \\
Intermediate & $(55-70]$ & 3 \\
Hard & $(25-50]$ & 4 \\
\hline
\end{tabular}

With these assumptions, we generate several scenarios corresponding to damage of different severity at different locations and extract the synthetic response of the model in the form of acceleration signals that can then be processed through AFDD to provide the modal properties (natural frequencies and mode shapes). The undamaged condition is swept with the temperature model. Each damage scenario starts from an undamaged 
model corresponding to certain temperature from which elastic modulus of the elements associated to the damaged region are reduced.

Once these models are created, synthetic acceleration signals are generated by applying a white noise excitation and obtaining the responses at the same places where the sensors were located in the real structure. The AFDD algorithm is used to process these time series to extract natural frequencies and mode shapes as if it were real experimental input. The final damage sensitive features to feed the SVM algorithm are the first two first bending eigenfrequencies and MAC values.

\section{c) Configuration of the SVM algorithm}

SVM is a data-driven algorithm to solve classification and regression problems [20]. It learns relationships in the data and finds the optimal space to separate the data among the different classes [21]. This approach results to be very versatile since it can fit many different types of discriminant functions from which the hypothetic classification space is formed, including linear, neural network or radial basis models [23]. Particularly, this algorithm can be understood as an optimization problem in the sample space with the following formulation [23]:

$$
\begin{aligned}
& \underset{\beta_{0}, \beta}{\min }\|\beta\|_{2}^{2} \\
& \text { subject to } y_{i}\left(x_{i}^{T} \beta+\beta_{0}\right) \geq 1 \quad \forall i=1, \ldots, n,
\end{aligned}
$$

where $\boldsymbol{x}_{i}$ is a feature vector in the sample space, $\beta$ is the slope of a normal to the optimal separating hyperplane, $\beta_{0}$ is an intercept, and $y_{i}$ is a class variable.

SVM technique is usually applied to solve two-class classification problems (the class variable is then binary and can take values of either +1 or -1 ), although it can also be extended to multi-class problems [23]. In our case, we aim at classifying between four possible severities of damage, being undamaged, slight damage, intermediate damage and hard damage.

The configuration of the algorithm implies two main stages: training and validation. Therefore, we generate a synthetic training dataset to feed the algorithm during training and correspondingly a testing dataset to validate the algorithm and check its performance.

The training set contains labelled information (damage sensitive features and corresponding class variable value) for the SVM algorithm to learn from that information and find its parameter values. Specifically, this dataset contains several scenarios associated to each of the classes to be identified in order to adequately characterize each group.

It is important to mention that, although damage at differentiated regions of the bridge is generated, the class assignation only depends on the severity of the induced damaged, that is, damage at two different regions with the same severity correspond to the same class. Hence, the class variable is an integer value in the range $[0,3]$, where 0 corresponds to undamaged, and 3 corresponds to hard damage. 
The next step is validation. We feed the algorithm with new scenarios (unseen by the algorithm during training) whose class variable is known. The trained SVM will receive these new cases and predict the class to which they correspond, based on the training. We calculate the performance of the algorithm comparing the predictions on the SVM and the real structural condition associated to each element in the validation dataset:

$$
\text { Performance }(\%)=\frac{\text { number of correct predictions }}{\text { size of testing dataset }} \times 100
$$

\section{Results}

In this section, we present the results obtained from the application of the SVM algorithm following the steps presented in the previous section. In order to show the results graphically, we applied Principal Component Analysis (PCA) to reduce the dimensions of the feature space to $2 \mathrm{D}$ and thus make it representable. Fig. 7 shows the results including both training and validation datasets, where the colored regions correspond to each class to be classified (from left to right, increasing severity of damage, indicated by the class variable number). The performance of the algorithm is calculated for the validation dataset, providing an $87 \%$ according to equation (9) that is indeed a good result.

In addition, we observe that mainly for the hard damage scenario, the different regions where damage exist are easily distinguished. Hence, for strong damages, the algorithm also informs on the possible location of this damage, suggesting further inspections in that region. The effect of temperature variations was considered as undamaged scenarios. It also induces certain variability in the different damage scenarios, but it does not generate conflict in the assignation of the damage severity.

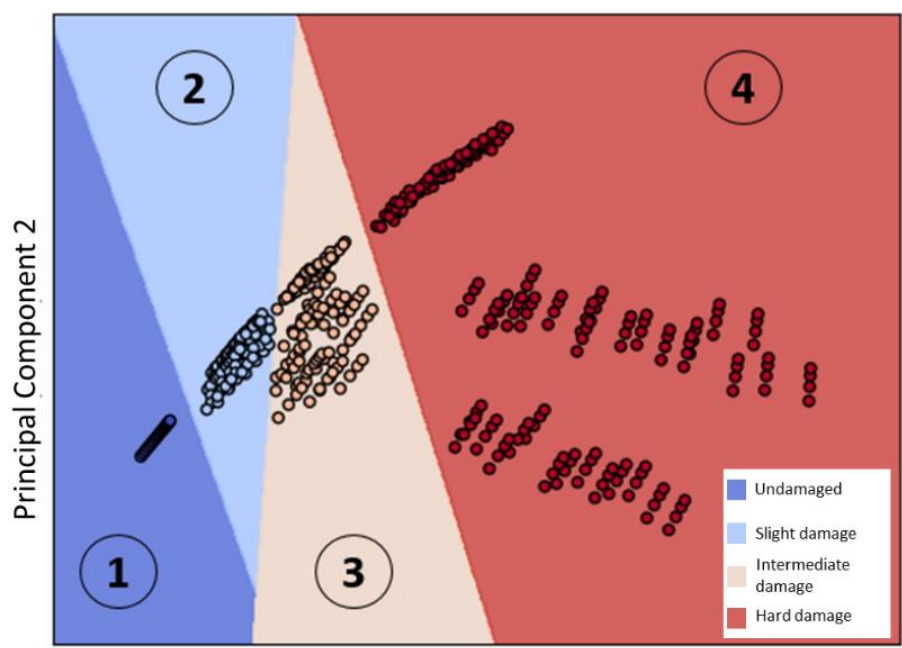

Principal Component 1

Fig. 7 2D representation of the SVM results with four damage classes 


\section{Conclusions and future work}

This work addresses the implementation of a damage detection tool based on a machine learning algorithm known as Support Vector Machine, using the modal response of a truss bridge. The work starts from the calibration of a FE model of the bridge by submitting it to an updating procedure that minimizes the discrepancy between the experimental and the numerical responses. These responses are obtained by applying the AFDD algorithm to acceleration measurements. Next, the calibrated FE model is employed to generate multiple scenarios to train and validate the data-driven algorithm.

We account for the environmental variability by relating the elastic modulus of the steel elements of the bridge with ambient temperature, thus allowing to sweep a wide range of undamaged service conditions. The presence of damage is represented by localized reductions of the elastic modulus depending on the degree of severity of damage. 4 levels of damage are considered for the SVM algorithm to solve the classification problem, being undamaged, slight damage, intermediate damage and hard damage. Based on these assumptions, the training and validation datasets are synthetically generated and fed to the SVM algorithm to model the classifier. Results show that the algorithm generates predictions with a performance close to $90 \%$, thus suggesting its implementation for evaluating new data in future monitoring processes.

Since SVM rapidly detects abnormal behavior, it can be used as a preliminary alert system to activate a more complex damage identification technique, such as FE model updating. In this strategy, once the alert is triggered by the SVM, the undamaged and current mode shapes can be used to perform an updating procedure based on the GA algorithm of the calibration step. That is, the alert system controls the correct performance of the structure in real time and, if novelty is detected, an exhaustive damage identification (not real-time) is conducted through model-based techniques.

\section{$5 \quad$ Acknowledgements}

This project has received funding from the European Union's Horizon 2020 research and innovation program under the grant agreement No 769373 (FORESEE project). This paper reflects only the author's views. The European Commission and INEA are not responsible for any use that may be made of the information contained therein.

\section{$6 \quad$ References}

1. Farrar CR. Structural Health Monitoring: a Machine Learning Perspective. 2013.

2. Huston D. Structural Sensing, Health Monitoring and Performance Evaluation. Taylor \& Francis; 2011.

3. Brownjohn JMW, de Stefano A, Xu YL, Wenzel H, Aktan AE. Vibration-based monitoring of civil infrastructure: Challenges and successes. Journal of Civil Structural Health Monitoring 2011; 1(3-4): 79-95. DOI: 10.1007/s13349-011-0009-5.

4. Soe MT. Vibration-Based Finite Element Model Updating and Structural Damage Identification 2013(May). 
5. Brincker R, Ventura C. Introduction to Operational Modal Analysis. 2015.

6. Reynders E, Houbrechts J, De Roeck G. Fully automated (operational) modal analysis. Mechanical Systems and Signal Processing 2012; 29: 228-250. DOI: 10.1016/j.ymssp.2012.01.007.

7. Carden EP, Fanning P. Vibration based condition monitoring: A review. Structural Health Monitoring 2004; 3(4): 355-377. DOI: 10.1177/1475921704047500.

8. Brincker R, Zhang L, Andersen P. Modal identification of output-only systems using frequency domain decomposition. Smart Materials and Structures 2001; 10(3): 441. DOI: 10.1088/0964-1726/10/3/303.

9. Brownjohn JMW, Magalhaes F, Caetano E, Cunha A. Ambient vibration re-testing and operational modal analysis of the Humber Bridge. Engineering Structures 2010; 32(8): 2003-2018. DOI: 10.1016/j.engstruct.2010.02.034.

10. Magalhães F, Cunha Á. Explaining operational modal analysis with data from an arch bridge. Mechanical Systems and Signal Processing 2011; 25(5): 1431-1450. DOI: 10.1016/j.ymssp.2010.08.001

11. Brincker R, Andersen P, Niels-Jorgen J. Automated Frequency Domain Decomposition for Operational Modal Analysis.

12. Magalhães F, Cunha Á, Caetano E. Online automatic identification of the modal parameters of a long span arch bridge. Mechanical Systems and Signal Processing 2009; 23(2): 316 329. DOI: 10.1016/j.ymssp.2008.05.003.

13. Friswell MI, Mottershead JE. Finite Element Model Updating in Structural Dynamics. vol. 38. Dordrecht: Springer Netherlands; 1995. DOI: 10.1007/978-94-015-8508-8.

14. Vitola J, Tibaduiza D, Anaya M, Pozo F. Structural Damage detection and classification based on Machine learning algorithms. 8th European Workshop On Structural Health Monitoring 2016(July): 5-8.

15. Mujica LE, Rodellar J, Fernández A, Güemes A. Q-statistic and t2-statistic pca-based measures for damage assessment in structures. Structural Health Monitoring 2011; 10(5): 539-553. DOI: $10.1177 / 1475921710388972$.

16. Ding Y, Li A. Assessment of bridge expansion joints using long-term displacement measurement under changing environmental conditions. Frontiers of Architecture and Civil Engineering in China 2011; 5(3): 374-380. DOI: 10.1007/s11709-011-0122-x.

17. Rytter A. Vibrational Based Inspection of Civil Engineering Structures 1993.

18. Bakdi A, Kouadri A, Mekhilef S. A data-driven algorithm for online detection of component and system faults in modern wind turbines at different operating zones. Renewable and Sustainable Energy Reviews 2019; 103(January 2018): 546-555. DOI 10.1016/j.rser.2019.01.013.

19. Chen HP. Structural Health Monitoring of Large Civil Engineering Structures. Wiley Blac. Wiley Black; 2018. DOI: 10.1002/ejoc.201200111.

20. Worden K, Manson G. The application of machine learning to structural health monitoring. Philosophical Transactions of the Royal Society A: Mathematical, Physical and Engineering Sciences 2007; 365(1851): 515-537. DOI: 10.1098/rsta.2006.1938.

21. HoThu H, Mita A. Damage Detection Method Using Support Vector Machine and First Three Natural Frequencies for Shear Structures. Open Journal of Civil Engineering 2013; 03(02): 104-112. DOI: 10.4236/ojce.2013.32012.

22. Meruane V, Heylen W. Structural damage assessment under varying temperature conditions. Structural Health Monitoring 2012; 11(3): 345-357. DOI: 10.1177/1475921711419995.

23. Sen D, Nagarajaiah S. Data-Driven Approach to Structural Health Monitoring Using Statistical Learning Algorithms 2018: 295-305. DOI: 10.1007/978-3-319-68646-2_13. 